\title{
Indicação da anuscopia de alta resolução e citologia anal na prevenção de HPV e câncer colorretal em pacientes portadores de HIV
}

\section{Indication of high resolution anoscopy and anal cytology for prevention of HPV and colorectal cancer in HIV patients}

\author{
HUGO LEONARDO MADEIRO ARCANJO SILVA ${ }^{1}$; LEANDRO VALÉRIO COSTA BATISTA ${ }^{1}$; \\ LUCIANE DE LIMA MOURA ${ }^{1}$; LUIIS CARLOS VIEIRA TAVARES JÚNIOR ${ }^{1}$; JULIANA AROUCHA ${ }^{2}$; \\ SANDRA GICO BELO ${ }^{3}$, MANOEL ÁLVARO LINS NETO ${ }^{4}$
}

\begin{abstract}
${ }^{I}$ Doutorandos do curso de Medicina da Universidade Federal de Alagoas (UFAL) - Maceió (AL), Brasil; ${ }^{2}$ Patologista do Hospital Universitário Professor Alberto Antunes da UFAL; Staff do Serviço de Coloproctologia do Hospital Universitário Professor Alberto Antunes da UFAL - Maceió (AL), Brasil.; ${ }^{3}$ Coloproctologista do Serviço de Coloproctologia do Hospital Universitário Prof. Alberto Antunes (HUPAA/UFAL) ${ }^{4}$ Professor Adjunto e Chefe do Serviço de Coloproctologia do Hospital Universitário Professor Alberto Antunes da UFAL - Maceió (AL), Brasil.
\end{abstract}

SILVA HLMA, BATISTA LVC, MOURA LDL, JÚNIOR LCVT, AROUCHA J, BELO SG, NETO MÁL. Indicação da anuscopia de alta resolução e citologia anal na prevenção de hpv e câncer colorretal em pacientes portadores de hiv. Rev bras Coloproct, 2011;30(4): 393-398.

RESUMO: Objetivo: Rastreamento do papilomavírus humano (HPV) assim como sua correlação com a neoplasia anorretal nos pacientes portadores do vírus da imunodeficiência humana (HIV) por meio da anuscopia de alta resolução (AAR) e da citologia anal. Métodos: Desenvolvemos um estudo observacional, transversal e duplo-cego em que participaram 31 pacientes portadores do HIV independente de queixas proctológicas. Os pacientes foram submetidos à AAR e citologia anal cujas lâminas foram enviadas ao setor de anatomia patológica para coloração e posterior análise. Resultados: Verificamos 22 pacientes do sexo masculino e 9 do sexo feminino entre 20 e 67 anos. Dos 31 analisados, quatro encontravam-se em estágio de imunodepressão, 23 utilizavam terapia antirretroviral, 16 com passado de sexo anal receptivo e 12 com passado de condiloma acuminado. À AAR 11 pacientes tinham alterações e 7 foram confirmados pela citologia. Verificamos ainda oito pacientes com alterações à citologia os quais possuíam ausência de alterações à AAR. À citologia apresentaram três células escamosas atípicas de significância (Ascus, do inglês atypical squamous cells of undetermined significance), cinco com paraceratose e/ou hiperceratose, seis lesões intraepiteliais de baixo grau e uma lesão intraepitelial de alto grau. Conclusão: Sugerimos a utilização da AAR aliada à citologia anal para rastreamento nos pacientes portadores do HIV, visto que esses exames complementam-se para a deteç̧ão de lesões que estejam relacionadas ao câncer anorretal.

Palavras-chave: Papilomavirus. Neoplasia anal. HIV.

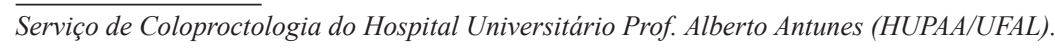

$\overline{\text { Recebido em 04/09/2010 }}$

Aceito para publicação em 27/10/10 
Rev bras Coloproct

Outubro/Dezembro, 2010
Indicação da anuscopia de alta resolução e citologia anal na prevenção de HPV e câncer colorretal em pacientes portadores de HIV

Hugo Leonardo Madeiro Arcanjo Silva e Cols.
Vol. 30

$\mathrm{N}^{\circ} 4$

\section{INTRODUÇÃO}

A infecção pelo papilomavírus humano (HPV) é a doença sexualmente transmissível mais frequente no mundo. No Brasil, o Ministério da Saúde constatou $23,4 \%$ nas doenças sexualmente transmissíveis (DST) e registra a cada ano 137 mil novos $\operatorname{casos}^{1,2}$. Diversos estudos ${ }^{3,4}$ mostram consistentemente uma alta prevalência e incidência do HPV em homens e mulheres portadores do vírus da imunodeficiência humana (HIV), junto com lesões precursoras de câncer, como neoplasia intraepitelial cervical (NIC) e neoplasia intraepitelial anal (NIA) particularmente entre os homens HIV positivos que praticam sexo com homens (HSH). Nos HSH nãoinfectados pelo HIV foi demonstrada uma incidência de câncer de ânus de 35/100 mil, e em HSH HIV positivos tem sido sugerido um valor duas vezes maior, de 70/100 mil, como consequência da alta prevalência de infecção por tipos oncogênicos de papiloma vírus ${ }^{5}$.

Hoje são conhecidos mais de 100 subtipos de HPV, destes, 30 possuem tropismo pelo trato anogenital, e são divididos em dois grupos de acordo com o seu potencial de oncogenicidade. Os de baixo risco, os tipos 6 e 11 são os mais prevalentes, assim como os de alto risco oncogênico, por exemplo, os HPV 16 e $18^{6}$. O vírus, por si só, não é suficiente para a carcinogênese, e a progressão tumoral que ocorre em pequena porcentagem de indivíduos pode ser estimulada por mutágenos químicos ou físicos ${ }^{7}$.

Assim como acontece com a NIC, o condiloma acuminado do trato genital inferior, a NIA e a vulvar são muito mais comuns nas mulheres HIV positivas do que nas HIV negativas. Um recente estudo prospectivo de coorte encontrou a preexistência de condiloma acuminado no trato genital inferior ou neoplasia intraepitelial vulvar e anal em 6\% de 481 mulheres HIV positivo comparado com $1 \%$ de 437 mulheres HIV negativo $(p<0,001)^{8,9}$.

O objetivo do presente estudo foi verificar a presença do vírus HPV em suas diversas formas, condiloma acuminado e subclínica, e sua correlação com a neoplasia anorretal nos pacientes portadores do vírus HIV atendidos no Hospital Dia Infectologia da Universidade Federal de Alagoas (UFAL).

\section{PACIENTES E MÉTODOS}

Trata-se de um estudo observacional, transversal e duplo-cego em que foram estudados 31 pacientes portadores do HIV, de ambos os sexos, os quais foram atendidos no Ambulatório de Coloproctologia do Hospital Universitário Professor Alberto Antunes da UFAL, no período de abril de 2008 a dezembro de 2009. Este trabalho foi submetido e aprovado pelo Conselho de Ética da UFAL.

Os critérios de inclusão para os pacientes foram os seguintes: idade maior que 18 anos; ser portador do HIV, independentemente do estadiamento da doença; concordado em participar da pesquisa, após a explicação do protocolo de pesquisa e assinado o termo de consentimento livre e esclarecido.

Os dados foram obtidos por meio do preenchimento de um protocolo de pesquisa, sob a forma de uma entrevista clínica estruturada, sempre com os mesmos entrevistadores. Após essa etapa, os pacientes foram submetidos ao exame proctológico, em decúbito lateral esquerdo (posição de Sims), utilizando um aparelho de colposcopia, sendo essa fase denominada de anuscopia de alta resolução. Numa primeira etapa foi realizada coleta de material para citologia oncótica, sendo confeccionadas duas lâminas (uma externa - pele perianal; e outra interna - mucosa anorretal) e usado fixador para conservação do material obtido. $\mathrm{O}$ próximo passo consistiu na aplicação de ácido acético a $5 \%$ na pele e na mucosa anorretal por dois minutos, retirando-se o excesso do ácido com soro fisiológico, a seguir aplicou-se o azul de toluidina, após essa etapa foram investigadas as lesões através do colposcópio.

O material coletado foi enviado para o setor de Anatomia Patológica do Hospital Universitário Professor Alberto Antunes da UFAL para realização da citologia oncótica e analisada pela patologista Dra. Juliana Aroucha, que não possuía informações prévias sobre o resultado da anuscopia de alta resolução (AAR).

Os pacientes foram orientados a retornar ao Ambulatório de Coloproctologia após um mês, para definição diagnóstica e tratamento quando necessário.

\section{RESULTADOS}

Entre os 31 pacientes estudados, 22 eram do sexo masculino (70,9\%) e 8 do feminino (29\%). A média de 
Rev bras Coloproct

Outubro/Dezembro, 2010
Indicação da anuscopia de alta resolução e citologia anal na prevenção de HPV e câncer colorretal em pacientes portadores de HIV

Hugo Leonardo Madeiro Arcanjo Silva e Cols.
Vol. 30 idade foi de 37,9 anos, variando de 20 a 67 anos. Durante o período da coleta, 4 pacientes $(12,9 \%)$ possuíam contagem de linfócitos CD4+ $\leq 200$ células $/ \mathrm{mm}^{3}, 3$ do sexo masculino e 1 feminino, e 27 pacientes $(87,1 \%)$ apresentavam CD4 superior 200 células $/ \mathrm{mm}^{3}$ (Tabela1). Vinte e três pacientes $(74,2 \%)$ utilizavam terapia antirretroviral combinada (TARV). A opção sexual foi indagada, 29\% dos pacientes declararam-se homossexuais, $64,5 \%$ heterossexuais e $6,5 \%$ bissexuais; 17 $(54,84 \%)$ relataram ter praticado atividade sexual anal receptiva (Tabela 2). Passado de condiloma foi questionado e 12 pacientes $(38,7 \%)$ declararam positividade, enquanto 19 pacientes $(61,3 \%)$ não relataram.

Durante o exame de AAR, com magnificação de imagem através do colposcópio e utilizando a reação com ácido acético a 5\%, observaram-se 11 reações cetoácidas positivas $(35,5 \%)$, ou seja, apresentou coloração esbranquiçada na região analisada. Nesse grupo, um voluntário possuía CD4 $+\leq 200$. Vinte pacientes foram não-reagentes $(64,5 \%)$, dos quais 17 apresentavam valores do CD4+ acima de 200 células $/ \mathrm{mm}^{3}$ $(54,8 \%)$ e 3 com valores de CD4 $<200$ células $\mathrm{mm}^{3}$ ou seja $9,7 \%$ (Tabela 3 ).

Após a leitura das lâminas com os espécimes coletados da região anorretal, obtivemos os seguintes resultados: 3 (9,7\%) apresentavam células do epitélio estratificado com significado indeterminado (Ascus) (os pacientes possuíam CD4+>200); 5 apresentavam células com paraceratose e/ou hiperceratose (16,1\%); destes, $1(3,2 \%)$ apresentava $\mathrm{CD} 4+\leq 200 ; 6$ pacientes $(19,4 \%)$, apresentavam lesões intraepiteliais de baixo grau na classificação de Bethesda de 1988; um deles (3,2\%), com valor imunossuprimido de linfócitos. Um paciente apresentou lesão intraepitelial de alto grau com CD4 >200 (Tabela 4).

Foram verificadas anormalidades na leitura das lâminas de 15 pacientes $(48,4 \%)$ e 16 pacientes $(51,6 \%)$ não apresentaram alteração citológica.

Houve concordância dos resultados da citologia e da AAR em 67,7\% e discordância em 32,3\% (Tabela 5).

Dos $15(48,4 \%)$ pacientes que apresentaram alterações citológicas anorretais, 7 (46,7\%) apresentavam lesões intraepiteliais de baixo ou alto grau.

Pudemos evidenciar que dos 17 pacientes que haviam praticado sexo anal receptivo, $41,1 \%$ apresentaram alteração citológica. Entre os pacientes que negaram praticar coito anal receptivo, $57,1 \%$ apresentavam anormalidade na citologia (Tabela 6).
Tabela 1 - Distribuição dos pacientes de acordo com contagem de linfócitos CD4

\begin{tabular}{lcr}
\hline Contagem de CD4 & n & \% \\
\hline$\leq 200$ & 4 & 12,9 \\
$>200$ & 27 & 87,1 \\
Total & 31 & 100,0 \\
\hline
\end{tabular}

Tabela 2 - Distribuição dos pacientes de acordo com a prática de atividade sexual anal receptiva

\begin{tabular}{lcc}
\hline Atividade sexual anal receptiva & $\mathbf{n}$ & $\mathbf{\%}$ \\
\hline Sim & 17 & 54,8 \\
Não & 14 & 45,2 \\
Total & 31 & 100,0 \\
\hline
\end{tabular}

Tabela 3 - Distribuição dos pacientes de acordo com resultado da reação com ácido acético a 5\%

\begin{tabular}{lcr}
\hline $\begin{array}{l}\text { Resultado da reação com ácido } \\
\text { acético a 5\% }\end{array}$ & n & \% \\
\hline Positiva & 11 & 35,5 \\
Negativa & 20 & 64,5 \\
Total & 31 & 100,0 \\
\hline
\end{tabular}

Tabela 4 - Distribuição dos pacientes de acordo com resultado da citologia anal

\begin{tabular}{lcc}
\hline Resultado da citologia & n & \% \\
\hline Sem alterações & 16 & 51,6 \\
Epitélio estratificado com & 3 & 9,7 \\
significado indeterminado & & 16,1 \\
Paraceratose e/ou hiperceratose & 5 & 19,4 \\
Lesão intraepitelial de baixo grau & 6 & 3,2 \\
Lesão intraepitelial de alto grau & 1 & 100 \\
Total & 31 & \\
\hline
\end{tabular}

Tabela 5 - Relação entre os Resultados da Citologia e da Anuscopia de Alta resolução

\begin{tabular}{lcc}
\hline & $\begin{array}{c}\text { Citologia } \\
\text { alterada }\end{array}$ & $\begin{array}{c}\text { Citologia } \\
\text { normal }\end{array}$ \\
\hline Anuscopia alterada & 8 & 3 \\
Anuscopia normal & 7 & 13 \\
\hline
\end{tabular}


Rev bras Coloproct

Outubro/Dezembro, 2010
Indicação da anuscopia de alta resolução e citologia anal na prevenção de HPV e câncer colorretal em pacientes portadores de HIV

Hugo Leonardo Madeiro Arcanjo Silva e Cols.
Vol. 30

$\mathrm{N}^{\circ} 4$
Tabela 6 - Relação entre o relato da prática do coito anal receptivo com a presença de alteração citológica

\begin{tabular}{lcc}
\hline \multirow{2}{*}{ Coito anal receptivo } & \multicolumn{2}{c}{ Alteração na citologia } \\
\cline { 2 - 3 } & Presente & Ausente \\
\hline Sim & 7 & 10 \\
Não & 8 & 6 \\
\hline
\end{tabular}

\section{DISCUSSÃO}

Por meio deste trabalho buscamos verificar a presença do HPV nas suas diversas formas e a sua associação com a neoplasia anorretal nos pacientes portadores do HIV, visto que essa relação já foi fortemente elucidada em estudos anteriores.

Nossa casuística compõe-se de 31 pacientes portadores de HIV com faixa etária de 20 a 67 anos de idade, média de 37,9 anos, com uma predominância do sexo masculino e a maioria heterossexual, confirmou a tendência atual em relação à faixa etária e opção sexual, mas contrariamente à feminilização do $\mathrm{HIV}^{10}$.

Diversos estudos ${ }^{11-17}$ mostram que o coito anal receptivo apresenta correlação com citologias anais apresentando lesões intra-epiteliais, seja de baixo ou alto grau, porém o nosso estudo não apresentou esta correlação. Acreditamos que essa discordância tenha ocorrido devido à omissão desse dado por alguns pacientes do nosso estudo.

Diversos pesquisadores ${ }^{18-21}$ mostraram em seus trabalhos que a incidência do câncer anal pode aumentar paradoxalmente em pacientes infectados pelo HIV usando terapia antirretroviral (TARV), devido ao fato de os indivíduos aumentarem a sua expectativa de vida pelo tratamento; assim, aqueles com a infecção por HPV podem com o tempo desenvolver lesões precursoras de câncer anal, como a neoplasia intraepitelial anal de alto grau e, não sendo tratados, podem progredir para o carcinoma epitelial anal. Neste estudo constatamos que sete pacientes apresentaram lesões intraepiteliais de baixo ou alto grau na citologia; destes, cinco participantes $(71,4 \%)$ faziam uso de TARV. Apesar dos dados acima concordarem com a literatura exposta, não podemos confirmar a relação HPV e NIA, pois em nosso estudo não houve um período de acompanhamento necessário para constatação dessa ocorrência. Contudo, a condução desses pacien- tes deve ser rigorosa, pelos diversos fatores já sabidos implicados na evolução da neoplasia anorretal.

Atualmente, estudos citológicos e com AAR têm detectado a presença do HPV em inúmeras outras formas de apresentação, nem sempre visíveis a olho nu, essas são chamadas formas subclínicas. Foi demonstrado alta sensibilidade de $98 \%$ da citologia em detectar lesões intraepiteliais anorretais precursoras cancerígenas comparada à biópsia; porém, a especificidade foi baixa, $50 \%$. Em nosso estudo, a concordância dos resultados da citologia e da AARAfoi de $64,5 \%$ e a discordância foi de $35,5 \%$. Em outros estudos, a AAR conseguiu detectar em $100 \%$ dos casos a infecção subclínica do HPV, bem como neoplasias intraepiteliais, constatados pela biópsia dirigida ${ }^{23}$. Semelhante ao que ocorre no câncer cervical, a citologia anal tem sido proposta para detecção das lesões precursoras da neoplasia anorretal, devendo ser incluída na rotina de cuidados dos pacientes portadores do HIV.

Neste estudo, a AAR apresentou-se positiva em $35,5 \%$ dos pacientes, dos quais $63,6 \%$ foram concordantes com a citologia anal. Por outro lado, em 64,5\%, a AAR foi negativa, dentre estes, a citologia conseguiu evidenciar lesões intraepiteliais de alto grau (LIEAG) ou baixo grau (LIEBG) em 15\% dos exames. Resultado esse relativamente discordante do encontrado por Nadal et al. ${ }^{24}$, no qual a AAR mostrou-se alterada em 51,7\%; levando em consideração esse percentual, em $69 \%$, a citologia foi concordante com a anuscopia e entre as AAR negativas (48,7\%), o exame citológico detectou LIEAG ou LIEBG em 40,7\% destes. Magi et al. ${ }^{22}$ trabalharam com pacientes HIV negativos e relataram positividade na AAR em $70 \%$ destes, onde todos foram confirmados pela citologia. Levando-se em consideração o número da amostra de cada trabalho, a análise crítica dependente do examinador e das possíveis lesões inflamatórias inespecíficas presentes em cada paciente, as quais podem gerar reação cetoácida positiva na análise da $\mathrm{AAR}^{25}$. Podemos inferir que os fatores acima citados, possivelmente, contribuíram para os diferentes achados.

Diante dos resultados encontrados em nosso trabalho e em consonância com a literatura podemos sugerir que: nos casos de citologia positiva com exame de AAR discordante, deve-se atentar para hipótese de possíveis lesões existentes no interior das criptas anais, as quais não são detectadas pela AAR, entretanto poderiam ser reveladas pela coleta e análise do ma- 
terial por meio da citologia ${ }^{5}$; e que nesses casos de discordância, deve-se manter elevada suspeição e nova avaliação deverá ser realizada em curto intervalo de tempo, de 6 a 12 meses após exame inicial ${ }^{5}$. Devemos sempre que possível realizar AAR, pois por meio deste procedimento poderemos realizar biópsias dirigidas ${ }^{13}$, visto que o aumento da incidência do câncer anal está comprovadamente associada à infecção pelo HPV, assim como ocorre com o câncer de útero ${ }^{6}$. Logo, existe crescente urgência de considerar o rastreamento e tratamento de NIA de alto grau para impedir a progressão dessa neoplasia em populações de risco.

\section{CONCLUSÃO}

A prevalência de alterações na AAR em pacientes portadores do HIV foi de $35,5 \%$. Já a citologia anal mostrou-se alterada em $48,4 \%$ dos pacientes. Houve concordância entre os exames de $64,5 \%$ e discordância em 35,5\%. Ao exame citológico foi evidenciada, em $22,6 \%$ dos pacientes, a presença de lesões de baixo e alto grau.

Portanto diante dos resultados obtidos, apesar de uma amostra pequena podemos sugerir que é de grande valia o rastreio nesses pacientes utilizando a AAR aliada à citologia anorretal, pois esses exames complementam-se para a detecção de lesões que estejam relacionadas ao câncer anorretal. Além disso, o custo-benefício é imensurável para esses pacientes e, substancialmente, para o serviço de saúde pública conforme foi vivenciado com o carcinoma de colo de útero quando foi utilizado o exame de Papanicolau como rotina para rastreamento dessa afecção.

\begin{abstract}
Objective: Screening for HPV as well as its correlation with anorectal cancer in patients carrying the human immunodeficiency virus (HIV) through high-resolution anoscopy (HRA) and anal cytology. Methods: We developed an observational study, double-blind attended by 31 patients with HIV independent proctologic complaints. Patients underwent HRA and anal cytology slides were sent to which sector pathology staining and analysis. Results: We found 22 males and 9 females between 20 and 67 years. Of the 31 tested, 4 were in stage of immunosuppression, 23 were using antiretroviral therapy, 16 with history of receptive anal sex and 12 with a history of condyloma acuminata. In the HRA 11 patients had changes and 7 were confirmed by cytology. We also verified eight patients with abnormal cytology who had no changes to the HRA. In the atypical squamous cells of undetermined significance (Ascus) cytology had three, five with parakeratosis and/or hyperkeratosis, six intraepithelial lesions and a low grade squamous intraepithelial lesion high grade. Conclusion: We suggest the use of anoscopy high resolution combined with anal cytology for screening in patients with the HIV, since these examinations are complementary for the detection of lesions that are related to anorectal cancer.
\end{abstract}

Keywords: Papilomavirus. Anal neoplasia. HIV.

\section{REFERÊNCIAS}

1. Magi JC, Magi DAS, Reche LMC, Flavinha T, Carvalho GT. Anuscopia com exacerbação para diagnóstico de papilomavírus humano ano-retal na forma subclínica. Rev bras Coloproct. 2002;22(3):178-83.

2. Schiffman M, Castle PE. Human papillomavirus: epidemiology and public health. J Natl Cancer Inst Monogr. 2003;(31):14-9.

3. Nadal RS, Manzione CR. Citologia como método para detecção de lesões precursoras do carcinoma anal. Rev bras Coloproct. 2005;25(1):72-4.

4. Chin-Hong PV, Vittinghoff E, Cranston RD, Buchbinder S, Cohen D, Colfax G et al. Age-Specific prevalence of anal human papillomavirus infection in HIV-negative sexually active men who have sex with men: the explore study. J Infect Dis 2004;190(12):2070-6.

5. Coutinho, JRH. Rastreamento de lesões pré-neoplásicas do ânus: citologia anal e anuscopia de alta resolução: novas armas para prevenção. Rev. Col Bras Cir. 2006;33(5):311-7.
6. Amaral JC, Câmara MEBS, Morais PGM, Barros LDF; Lins Neto MAF. Associação de lesões anorretais em portadoras de infecção genital por HPV e neoplasia cérvico-uterina. Rev bras Coloproct. 2009;29(2):203-8.

7. Wieland U, Pfister H. Papilloma virus in human pathology. In: Gross GE, Barrasso R. Human papilloma virus infection: a clinical atlas. Ullstein Mosby GmbH \& Co. KG, Berlin/ Wiesbaden, 1997. p. 1-18.

8. Conley LJ, Ellerbrock TV, Bush TJ, Chiasson MA, Sawo D, Wright TC. HIV-1 infection and risk of vulvovaginal and perianal condylomata acuminata and intraepithelial neoplasia: a prospective cohort study. Lancet. 2002;359(9301): 108-13.

9. Ferenczy A, Coutlée F, Franco E, Hankins C. Human papillomavirus and HIV coinfection and the risk of neoplasias of the lower genital tract: a review of recent developments. CMAJ. 2003;169(5):431-4.

10. Revista do Sistema Único de Saúde do Brasil. Epidemiologia e Serviços de Saúde. Secretaria de Vigilância em Saúde; Ministério da Saúde do Brasil. 2005;14(2). 
11. Daling JR, Weiss NS, Hislop TG, Maden C, Coates RJ, Sherman KJ. Sexual practices, sexually transmitted diseases, and the incidence of anal cancer. N Engl J Med. 1987;317(16):973-7.

12. Goldie SJ, Kuntz KM, Weinstein MC, Freedberg KA, Welton ML, Palefsky JM. The clinical effectiveness and costeffectiveness of screening for anal squamous intraepithelial lesions in homosexual and bisexual HIV-positive men. JAMA. 1999;281(19):1822-9.

13. Goldstone SE, Winkler B, Ufford LJ, Alt E, Palefsky JM. High prevalence of anal squamous intraepithelial lesions and squamous-cell carcinoma in men who have sex with men as seen in a surgical practice. Dis Colon Rectum. 2001;44(5):690-8.

14. Palefsky JM, Holly EA, Hogeboom CJ, Berry JM, Jay N, Darragh TM. Anal cytology as a screening tool for anal squamous intraepithelial lesions. J Acquir Immune Defic Syndr Hum Retrovirol. 1997;14(5):415-22.

15. Panther LA, Wagner K, Proper J, Fugelso DK, Chatis,PA, Weeden W, et al. High Resolution anoscopy findings for men who have sex with men: inaccuracy of anal cytology as a predictor of histologic high-grade anal intraepithelial neoplasia and the impact of HIV serostatus. Clinical Infec Dis. 2004;38(10):1490-2.

16. Ryan DP, Compton CC, Mayer RJ. Carcinoma of the anal canal. N Engl J Med. 2000;342(11):792-800.

17. Tyring SK. Antiviral agents, vaccines, and immunotherapies. Clin Infec Dis. 2006;42:586.

18. Bower M, Palmieri C, Dhillon T. AIDS-related malignancies: changing epidemiology and the impact of highly active antiretroviral therapy. Curr Opin Infect Dis. 2006;19(1):14-9.

19. Frisch M, Biggar RJ, Engels EA, Goedert JJ; AIDS-Cancer Match Registry Study Group. Association of cancer with
AIDS-related immunosuppression in adults. JAMA. 2001;285:1736-45.

20. Palefsky JM, Holly EA, Ralston ML, Da Costa M, Bonner H, Jay N, et al. Effect of highly active antiretroviral therapy on the natural history of anal squamous intraepithelial lesions and anal human papillomavirus infection. J Acquir Immune Defic Syndr. 2001;28(5):422-8.

21. Palefsky J. Human papillomavirus-related disease in people with HIV. Current Opinion HIV AIDS. 2009;4(1):52-6.

22. Magi JC, Rodrigues MRS, Moreno WD, Fraga JBP, Costa ACL, Formiga GJS. A importância da AAR para o diagnóstico do papiloma vírus humano anorretal na forma subclínica, das lesões anais intraepiteliais e do carcinoma "in situ" anal. Rev Colégio Bras Cirur. 2004;31(1).

23. Mathews WC, Sitapati A, Caperna JC, Barber RE, Tugend A, Go U. Performance of anal cytology in a clinical setting when measured against histology and high-resolution anoscopy findings. J Acquir Immune Defic Syndr. 2004;37(5).

24. Nadal SR, Calore EE, Manzione CR, Arruda CN, Cha JD, Formiga FB, et al. Sensibilidade e especificidade da citologia anal com escova no diagnóstico das lesões clínicas provocadas pelo papiloma vírus humano, comparando uma com duas coletas. Rev bras Coloproct. 2009;29(3):297-302.

25. Chiao EY. Duration of anal human papillomavirus infection among immunocompetent women: clues to anal cancer epidemiology and possible prevention strategies. Clin Infec Dis. 2009;4(5)8:547-9.

Endereço para correspondência:

Manoel Álvaro Lins Neto

Rua Deputado José Lages, 350, apto. 501

CEP 57035-330

Maceió (AL), Brasil

E-mail: mlinsneto@gmail.com 